Meta

Journal des traducteurs

Translators' Journal

\title{
Le Bureau des traductions (1934-1977)
}

\section{Fikri Méléka}

Volume 22, numéro 1, mars 1977

Histoire de la traduction au Canada

URI : https://id.erudit.org/iderudit/003622ar

DOI : https://doi.org/10.7202/003622ar

Aller au sommaire du numéro

Éditeur(s)

Les Presses de l'Université de Montréal

ISSN

0026-0452 (imprimé)

1492-1421 (numérique)

Découvrir la revue

Citer cet article

Méléka, F. (1977). Le Bureau des traductions (1934-1977). Meta, 22(1), 57-65.

https://doi.org/10.7202/003622ar d'utilisation que vous pouvez consulter en ligne.

https://apropos.erudit.org/fr/usagers/politique-dutilisation/ 


\section{Le Bureau des traductions (1934-1977)}

UN ORGANISME QUI SE CONJUGUE AU PASSÉ PRÉSENT... ET AU PASSÉ FUTUR

Si l'on en croit la petite histoire, le Bureau des traductions serait le fruit du hasard... Plus exactement, il serait né de l'accès de mauvaise humeur d'un ministre, furieux et dépité de voir son autorité bafouée par la dizaine de traducteurs des Débats. C'était à l'occasion d'une conférence impériale sur le commerce, tenue à Ottawa en 1932. Le secrétaire d'État, M. C.H. Cahan, ayant demandé aux dits traducteurs d'assurer le service de traduction de la conférence, ceux-ci se récusèrent alléguant qu'ils ne travaillaient que pendant les sessions parlementaires. Et, effectivement, les traducteurs des Débats n'étaient de service que cinq ou six mois par an, le reste du temps ils étaient en congé... payé. Cahan dut donc faire appel à des indépendants et devint blême de colère, rapporte-t-on, lorsqu'il s'aperçut qu'un certain nombre de traducteurs parlementaires figuraient au nombre des pigistes ${ }^{1}$ !

D'autres affirment que l'idée de centraliser les services de traduction, donc de créer le Bureau, aurait germé dans l'esprit du tout-puissant greffier adjoint de la Chambre des communes, Arthur Beauchesne, soucieux de « tailler un poste intéressant pour l'un de ses amis, chef d'une unité de traduction, Hector Carbonneau.

Il convient toutefois de préciser que le projet de centralisation des services de traduction était déjà dans l'air depuis assez longtemps puisque le journal outaouais le Droit en parlait une dizaine d'années plus tôt. Il avait consacré un article à la question dans son numéro du 8 février 1924, et c'était (déjà) pour s'y opposer.

\section{Opposition unanime}

Quoi qu'il en soit, le projet de loi du secrétaire d'État prévoyant la création du Bureau des traductions se heurte, dès le début, à l'opposition unanime des Canadiens français. Les raisons ne sont cependant pas les mêmes pour tous. Les

1. Selon une autre version, la Chambre ne siégeait pas à ce moment et le secrétaire d'Etat n'avait pu mettre la main sur les traducteurs des Débats qui avaient déserté Ottawa pour la Côte d'Azur où, dit-on, ils avaient l'habitude de passer leurs congés sessionnels. 
hommes politiques, en majorité libéraux, y sont hostiles parce que l'initiative émane d'un gouvernement conservateur (cabinet Bennett) ${ }^{2}$. Les traducteurs le repoussent par crainte de perdre leur autonomie (chaque ministère ou organisme fédéral avait son propre service), en particulier les chefs qui se refusent à devenir des subalternes alors que, dans les ministères, ils sont rois et maîtres. L'Association technologique de langue française, groupement composé en majorité de traducteurs de la région d'Ottawa-Hull, se réunit et l'un de ses membres, Cinq-Mars, déclare, vivement applaudi par ses collègues : «Il y a péril en la demeure. \$ Les traducteurs parlementaires craignent, pour leur part, de ne plus bénéficier des privilèges inhérents à leur charge, à savoir six ou sept mois de congés sessionnels par an.

La presse francophone constitue le troisième pôle d'opposition. Elle n'est pas hostile, en général, au principe même de la centralisation des services de traduction mais réclame plutôt que le projet de loi s'accompagne d'un certain nombre de garanties, notamment l'assurance formelle que tous les documents fédéraux seront traduits (et rapidement) en français, que la spécialisation sera maintenue, que les unités de traduction dans les ministères seront accrues et qu'aucun traducteur ne sera renvoyé (en deux ans, vingt postes avaient été supprimés par l'abolition des services de traduction au ministère des Finances et à la Commission des chemins de fer et par la réduction du nombre de traducteurs au ministère de la Marine et à la Chambre des communes). Certains journaux accusent le gouvernement de chercher, grâce au subterfuge de la centralisation, à "parquer » les Canadiens français dans les services de traduction et à ne leur réserver au sein de la fonction publique fédérale que des postes de traducteur, tout en s'arrangeant de surcroît pour ne pas les multiplier.

Les média, qui déclenchent une véritable levée de boucliers, saisissent l'occasion pour reprocher au cabinet Bennett de ne pas se conformer aux dispositions de l'article 133 de l'Acte de l'Amérique du Nord britannique - une foule de rapports et de renseignements de tous ordres ne sont pas traduits ou, s'ils le sont, c'est avec beaucoup de retard, ce qui leur fait perdre toute utilité - dénoncer l'insuffisance des services de traduction et se plaindre que les déclarations des responsables du gouvernement et de l'administration, les communiqués et les documents importants ne sont fournis à la presse qu'en anglais, ce qui désavantage les journaux d'expression française et amène souvent leurs correspondants dans la capitale fédérale à s'improviser traducteurs.

\section{Attachés parlementaires}

Le cabinet Bennett réussit toutefois, malgré cette forte opposition, à faire adopter le projet de loi instituant le Bureau des traductions en tant que seul organisme gouvernemental chargé d'assurer, dans toutes les langues, les services de traduction nécessaires au bon fonctionnement de l'administration fédérale et des institutions qui en relèvent. Le Bureau est cré en février 1934 et sa direction

2. Seul Henri Bourassa, indépendant, défend le projet de loi estimant que la centralisation des services de traduction enracinerait l'usage du français dans la fonction publique fédérale. 
confiée à un surintendant nommé par la Commission de la fonction publique (appelée alors Commission du service civil), M. Damien-T. Robichaud, qui occupe d'abord le bureau de... Cahan à la Chambre, puis celui du conseiller juridique du Sénat avant de s'installer, toujours sur la colline parlementaire, dans l'édifice de l'Ouest où se trouvent toujours d'ailleurs certains services d'interprétation. Le surintendant est assisté d'un secrétaire, M. Georges Benoit, et d'un garçon de bureau. C'est là toute l'administration centrale. Il est vrai que tous les services d'intendance sont assurés par le ministère de tutelle, qui est le secrétariat d'État.

Bien que la traduction au Canada remonte à la conquête, ce lien avec le parlement n'est pas pour étonner. En effet, la traduction n'existait pas en tant que service gouvernementai fédéral avant la Confédération. Dès 1867, il fallut recruter des traducteurs pour les Débats et les Comités, les Pères de la Confédération ayant exigé qu'on conserve la langue française au Canada et que toutes les lois et tous les actes publics soient rédigés dans les deux langues officielles. Le Pacte de la Confédération garantit l'usage du français à Ottawa en même temps que l'anglais. C'est à ce moment qu'est née la traduction dans la capitale fédérale. En fait, les premières traductions n'ont lieu qu'en 1872-1873 et sont confiées à des pigistes. C'est au début du $\mathrm{XX}^{\oplus}$ siècle seulement que les traducteurs des Débats sont: titularisés et deviennent des fonctionnaires fédéraux.

Il n'est pas alors question d'affecter des traducteurs à tous les organismes gouvernementaux. Cette situation se perpétue assez longtemps puisqu'en 1913 il n'y a que sept ou huit traducteurs disséminés dans différents ministères.

$\mathrm{Au}$ moment de la création du Bureau, la fonction publique fédérale compte 91 traducteurs (au traitement global de 252000 dollars), dont une douzaine aux Débats. Le ministère des Affaires extérieures réussit, malgré les dispositions de la loi, à soustraire son service de traduction, alléguant le caractère confidentiel des documents traités. Il en est de même du Sénat. Les traducteurs des Affaires extérieures et du Sénat ne sont intégrés au Bureau qu'après la disparition de leurs chefs respectifs, soit en 1946 pour le ministère et en 1950 dans le cas du Sénat.

La tempête suscitée par le projet de loi sur la centralisation des services de traduction ne tarde pas à se calmer avec l'implantation du Bureau. Les oppositions s'atténuent rapidement et les réticences se font de moins en moins vives grâce à l'application intelligente des dispositions de la loi. Les unités ministérielles de traduction demeurent auprès du client et les craintes que certains avaient exprimées de voir tous les services réunis dans un même local, une sorte "d'usine de la traduction > comme on l'affirmait, sont sans fondement.

Sur le plan administratif, la création du Bureau n'affecte le secteur parlementaire, principal centre de cristallisation des réserves sinon d'opposition à l'initiative de Cahan, que de façon marginale. Les traducteurs cessent de relever de l'Orateur de la Chambre et passent sous la direction du surintendant du Bureau. Ils perdent toutefois (mais en partie seulement) le régime préférentiel dont ils bénéficiaient par rapport à leurs collègues. Auparavant, ils n'étaient tenus de travailler que pendant les sessions, c'est-à-dire cinq ou six mois par an. Un compromis est conclu avec la nouvelle direction qui leur accorde treize semaines de 
congé, dont dix sessionnelles. Les traducteurs des Comités obtiennent six semaines. Aujourd'hui encore, les interprètes et traducteurs parlementaires sont avantagés par rapport à leurs collègues des autres secteurs en raison des conditions particulières de leur travail.

Une autre conséquence de la centralisation est l'égalisation des salaires. Ceux-ci étaient fixés par l'orateur de la Chambre dans le cas des traducteurs parlementaires et par les ministères employeurs ou la Commission de la fonction publique pour les autres, d'où d'importants écarts en matière de rémunération.

Avant 1934, les nominations avaient souvent, surtout en ce qui concerne les Débats, un caractère politique. Il s'agissait tantôt de candidats malheureux aux élections, tantôt de journalistes, avocats ou autres membres de professions libérales, qu'on voulait « récompenser» ou tout simplement «caser». Certains avaient même, dit-on, leurs « nègres » qui travaillaient en leur lieu et place et auxquels ils versaient une partie du salaire qu'ils percevaient. Ces «nègres » étaient en général, ou avaient été, des journalistes.

\section{Gens de valeur et grands seigneurs}

Il faut dire que, recrutés par voie de concours ou nommés par complaisance ${ }^{3}$, les traducteurs sont tous gens de valeur : romanciers, essayistes, poètes, historiens, journalistes et, parfois même, de véritables érudits. Le choix n'était pas mauvais dans l'ensemble. Toute une pléiade d'écrivains canadiens réputés sont passés par le Bureau des traductions. Citons, au hasard, Louis Fréchette, Alfred Desrochers, Eva Sénécal, Alonzo Cinq-Mars, Benjamin Sulte, William Chapman, Louvigny de Montigny, Jules Fournier, François-Xavier Garneau, Robert Rumilly...

Ce sont aussi de grands seigneurs. D'aucuns se souviennent encore de l'époque (c'était avant la Seconde Guerre mondiale) où les traducteurs des Débats fréquentaient assidûment la promenade de la rue Sussex, à deux pas de la colline parlementaire, une cape nonchalamment jetée sur les épaules, un chapeau aux larges bords posé sur. la tête, une canne à pommeau d'ivoire, ostensiblement brandie d'une main gantée de noir, et échangeant, au gré des rencontres, des saluts plus ou moins cérémonieux.

\section{Recrutement difficile}

Avec la création du Bureau, le recrutement se fait de façon beaucoup plus stricte et par les soins exclusifs de la Commission de la fonction publique. Des annonces paraissent dans les journaux et des examens sont organisés tous les ans ou presque. On compte alors trois sortes d'examen selon le niveau de recrutement : traducteur principal (équivalant au réviseur dans le régime actuel), traducteur «senior» et traducteur «junior». Des examens de passage d'une classe à une autre ont également lieu.

À titre d'exemple de la difficulté des examens de recrutement, signalons qu'en 1947, sur 325 candidats, 15 seulement réussissent dont 12 avaient déjà une

3. Il faut se garder des généralisations car les nominations de ce genre n'étaient pas la règle. 
expérience en traduction. Ce qui signifie que la profession ne s'est enrichie que de trois éléments. Certains candidats se sont présentés six ou sept fois et l'on cite même le cas d'un traducteur qui ne fut admis qu'au terme de dix tentatives.

La correction des examens n'était pas, comme aujourd'hui, confiée à des spécialistes. Elle était effectuée par des membres de la Commission de la fonction publique ou des recteurs d'université qui, malgré tout leur savoir, ignoraient généralement tout de l'art et des techniques de la traduction.

Le Bureau créé, les ministères et organismes savent à qui s'adresser (ou peut-être commencent-ils à réaliser l'importance d'une profession qui venait de recevoir ses lettres patentes à défaut de titres de noblesse) et réclament, de plus en plus, des traducteurs. Les petites équipes travaillant sur place se multiplient assez rapidement malgré les difficultés de recrutement. Les plus importantes se trouvent à Statistique Canada, aux Transports et aux Lois. Les traducteurs qui ne sont pas affectés au parlement ou aux ministères sont regroupés au sein de la Traduction générale où l'on traduit des textes des Comités, les rapports des commissions royales d'enquête, les documents des ministères et organismes qui n'ont pas de service de traduction et le trop plein des équipes ministérielles. Les textes confiés à la Traduction générale comprennent également des directives, notes de service, correspondance, formulaires, discours, communiqués ainsi que des documents divers, en particulier des rapports annuels provenant d'organismes autonomes (Conseil national de recherches, Société centrale d'hypothèques et de logement, Radio-Canada, Gendarmerie royale). La division des Lois constitue un service « parlementaire » et est logée dans l'enceinte du corps législatif.

\section{Tradition familiale}

Pour quelques-uns de nos collègues, la traduction est une tradition familiale. On est traducteur de père en fils comme c'est souvent le cas chez les avocats, les notaires ou les médecins. Citons, à titre d'exemple, Alfred Garneau, fils de l'historien François-Xavier Garneau ; Léon Gérin, fils d'Antoine Gérin-Lajoie, qui était aussi historien ; Raymond Robichaud, chef actuel de la Division de l'interprétation et directeur de l'école des interprètes, fils du premier surintendant du Bureau, Damien-T. Robichaud ; Michel Gouin, jeune recrue de la Section scientifique centrale, fils de Jacques Gouin, ancien chef de la section de la Défense nationale.

Les besoins en traduction augmentent avec la guerre et certains ministères créent même leurs propres services, distincts du Bureau. C'est notamment le cas des Forces armées et du ministère des Approvisionnements et Services. Le Bureau, qui grossit rapidement, connaît une assez forte poussée après la fin des hostilités et, en 1948, il compte près de 175 traducteurs. Ce chiffre grimpe progressivement à 300 et se maintient longtemps à ce niveau. Le personnel, relativement plus âgé qu'aujourd'hui, est aussi beaucoup moins mobile que de nos jours.

La Loi sur les langues officielles (1960) amène le Bureau à intensifier le recrutement pour faire face à la demande sans cesse croissante des différents 
ministères et organismes, mais c'est en 1974 que l'embauchage atteint son point culminant avec près de 400 nouvelles recrues. Aujourd'hui, l'effectif comprend plus de 1850 personnes, dont les deux tiers sont des traducteurs et interprètes, le reste étant formé de personnel de gestion et de traitement de la copie. Une augmentation de près de $10 \%$ est prévue pour l'exercice 1977-1978.

\section{Nouveau mandat}

Un certain nombre de restructurations, comportant parfois une reclassification des postes, ont marqué l'histoire du Bureau. En 1974-1975, celui-ci entreprend une vaste opération de réorganisation, adaptée aussi bien à l'expansion de ses activités qu'à sa nouvelle vocation sur le plan de la terminologie, le Cabinet lui ayant confié la responsabilité de la normalisation terminologique, pour les deux langues officielles, au sein de l'administration fédérale.

Le Bureau, qui est le plus important employeur dans le domaine de la traduction au Canada, compte aujourd'hui cinq éléments : Direction générale du plan, Direction générale de la terminologie et de la documentation, Direction des opérations générales, Direction des opérations régionales et Direction des opérations spéciales.

\section{Planification et élaboration}

Constituée le $1^{\text {er }}$ novembre 1975 , la Direction générale du plan coiffe une direction de la planification et de l'élaboration des politiques (chargée de l'analyse de la conjoncture, de l'établissement des priorités, de l'évaluation de la performance, de la détermination des besoins et de la formulation des objectifs), une division de la qualité linguistique (dont le mandat consiste à veiller à la qualité des textes, notamment par l'appréciation des produits finis livrés aux clients) et une division de la formation. Celle-ci regroupe trois sections, chargées respectivement de l'initiation des traducteurs débutants, de la formation des réviseurs stagiaires et de l'application du programme de formation universitaire qui, par des bourses et des subventions à six universités agréées, fournit chaque année au Bureau une centaine de traducteurs diplômés.

La Direction du plan s'apprête à lancer un nouveau programme (formation des anglotropes) et envisage la création d'un Centre de communication destiné à servir de point focal de l'information interne et externe et à assurer la coordination des diverses publications existantes ou futures du Bureau.

Ce dernier publie actuellement l'Actualité terminologique, bulletin mensuel destiné aux spécialistes et dont le développement est à l'étude; les Bulletins de terminologie, lexiques consacrés à diverses disciplines qui s'adressent au même public, et Communication, organe de la direction à l'intention du personnel.

\section{Terminologie et documentation}

Le mandat de la Direction générale de la terminologie et de la documentation est triple : servir les besoins en terminologie du Bureau et des ministères et orga- 
nismes fédéraux, vérifier et normaliser la terminologie employée dans la fonction publique et établir, alimenter et exploiter une banque de données terminologiques, sur ordinateur, en collaboration avec les organismes provinciaux et privés qui s'intéressent à la recherche terminologique. La Direction a entrepris en septembre dernier l'installation, dans diverses sections opérationnelles, de terminaux destinés à répondre aussi bien aux besoins des traducteurs que des spécialistes des ministères clients. Elle a, d'autre part, établi de larges contacts aux niveaux fédéral, national et international dans le cadre de ses fonctions de normalisation et de liaison terminologiques.

Parallèlement à la mise sur pied du service de terminologie informatisé, un service analogue de documentation sera implanté d'ici trois ou quatre ans. Les textes traduits par les soins du Bureau seront classifiés et emmagasinés sur microfilms et fournis très rapidement aux traducteurs qui demandent des renseignements sur telle ou telle discipline. Des contacts ont lieu avec différents organismes au Canada et à l'étranger pour enrichir au maximum cette future banque documentaire.

\section{Trois directions opérationnelles}

Les trois autres directions ont, comme leur nom l'indique, des activités opérationnelles, c'est-à-dire la traduction des textes émanant de leurs clients. La Direction des opérations générales, la plus importante des trois, assure la traduction dans les deux langues officielles et, à l'occasion, des langues étrangères vers le français, pour le compte d'une centaine de ministères et d'organismes fédéraux à Ottawa. L'expansion de la demande l'a conduite à restructurer ses activités en réaménageant ses divisions qui, de trois, sont passées à sept, dont l'une centralise et coordonne les tâches de transcription des textes.

La Direction des opérations régionales fournit les mêmes services à l'extérieur de la région de la capitale nationale et dessert le ministère de la Défense nationale à Ottawa et dans les bases situées au Canada et en Allemagne occidentale (Lahr). Cet élément a considérablement accrû en 1975 ses activités à Montréal et à Québec dans le cadre de la politique de décentralisation géographique de l'administration fédérale. En conséquence, la Direction a créé deux divisions à Montréal et une à Québec, auxquelles s'ajoute la division de la Défense nationale et du Centre-Ouest. Elle comprend plusieurs sections spécialisées et dispose de son propre système d'initiation et de formation des débutants sous la forme de modules d'accueil. De plus, elle absorbe une bonne partie de l'excédent de la demande d'Ottawa.

La cinquième et dernière direction du Bureau est celle des opérations spéciales, qui fournit des services de traduction et d'interprétation au Parlement et à ses organismes constituants. Les services d'interprétation s'étendent également à tous les ministères et organismes publics ainsi qu'aux conférences multilingues et en langues officielles. Elle s'est dotée, en mai 1975, d'une très moderne école d'interprètes, destinée à lui assurer un recrutement sélectif. Treize stagiaires ont déja été formés et huit autres en suivent actuellement les cours. 
La Direction des opérations spéciales a absorbé, au début de 1976, deux éléments qui relevaient jusqu'alors des Opérations régionales : la division des services multilingues et la section des contrats. La première assure la traduction des langues étrangères (plus de 75) au français et à l'anglais. La seconde dispose d'un grand nombre de pigistes (plus de 350 ) auxquels elle confie les textes dont le Bureau ne peut assurer la traduction dans les délais requis.

\section{Nombreux projets à l'étude}

Plusieurs projets sont amorcés ou à l'étude. "L'opération client », destinée à rationaliser les rapports avec les ministères et organismes qui utilisent les services du Bureau, a été engagée en octobre dernier par une étude au niveau des unités de travail, s'est poursuivie par des visites aux clients et par trois journées d'étude réunissant les cadres de l'organisme et doit déboucher sur la rédaction d'une sorte de code définissant les relations entre les deux parties afin d'assurer le meilleur service possible à l'administration fédérale.

La Direction générale du plan poursuit l'étude de l'aménagement de la carrière des traducteurs, entreprise en septembre 1975 et qui pourrait aboutir à une refonte du plan de carrière, et envisage l'implantation de mécanismes de communication interne, comportant en particulier la création d'un périodique qui serait l'organe du personnel du Bureau.

\section{Traduction électronique}

Le Bureau a procédé, de septembre à décembre 1975, à des essais de traduction automatique de textes techniques, les seuls d'ailleurs qui puissent se prêter à la traduction électronique. C'est que le bilinguisme a suscité au Canada une demande considérable de traduction à laquelle on ne peut répondre par les moyens conventionnels. De nouvelles perspectives s'ouvrent ainsi aux traducteurs, dont certains seront probablement appelés à étudier l'informatique. De nouvelles spécialisations verront le jour et l'on aura, par exemple, des traducteurs-programmeurs, des traducteurs-analystes, etc.

Signalons enfin que le Bureau a été choisi, parallèlement à Statistique Canada et au ministère du Revenu national (Impôt), par le Conseil du Trésor pour servir de champ d'expérimentation à une nouvelle formule dite de la qualité de la vie au travail. Cette formule, essayée par le secteur privé en Europe occidentale, en Australie, aux États-Unis et au Canada, consiste à confier, sous certaines réserves touchant notamment à la classification, à la rémunération et au recrutement, la gestion de l'unité de travail au personnel qui la compose, sans préjudice naturellement au rendement quantitatif et qualitatif. Dans le cadre de la Q.V.T., deux sections du Bureau, regroupant chacune une vingtaine de personnes, s'administrent elles-mêmes depuis septembre 1976. Si l'expérience est concluante, la formule serait étendue à l'ensemble de la fonction publique fédérale.

L'ampleur prise ces dernières années par le Bureau des traductions et l'expansion de ses activités, jointes à sa nouvelle tâche de caractère national sinon 
international en matière de terminologie, amènent d'aucuns à s'interroger sur la vocation de cet organisme et à se demander si le temps n'est pas venu de le traiter en adulte et de voir en lui autre chose qu'un simple « programme » du secrétariat d'État. Et ils rappellent à ce sujet un projet conçu en 1961 par Noël Dorion, député de Bellechasse et secrétaire d'État dans le cabinet Diefenbaker, qui voulait lui donner ses lettres de noblesse et l'ériger au rang d'organisme majeur. La Presse du 9 novembre 1961 consacrait un article à ce projet sous le titre \& Le surintendant du Bureau des traductions deviendrait sous-ministre ».

L'histoire du Bureau des traductions serait incomplète si l'on ne disait quelques mots de l'Association technologique de langue française, ancêtre de l'actuelle Association des traducteurs et interprètes de l'Ontario (ATIO), qui regroupait la quasi-totalité du personnel du Bureau. Bien que juridiquement constituée le 2 mars 1921, l'Association technologique vit en fait le jour le 10 novembre 1920 à l'initiative de Louis d'Ornano, traducteur au ministère de la Marine. C'était une sorte d'académie du bon langage, où les traducteurs et « autres fonctionnaires fédéraux d'Ottawa faisant usage de la langue française $\gg$ se réunissaient pour discuter linguistique et stylistique. Les traducteurs et interprètes donnaient souvent lecture des lexiques et glossaires qu'ils constituaient dans le cadre de leur dur labeur. Bon nombre de ces modestes recueils furent publiés et constituèrent de précieux auxiliaires pour les jeunes traducteurs. Des causeries, fort intéressantes, animaient également ces rencontres. L'ATIO succéda à l'Association technologique le 10 septembre 1962 et prit un caractère plus nettement professionnel. Elle réunit aujourd'hui plusieurs centaines de traducteurs et interprètes, dont l'écrasante majorité est constituée par le personnel du Bureau

FiKRI MÉLÉkA 